\title{
Treatment and Handling of Purulent Core Production in Facial Spatial Infection by VSD Technique
}

\author{
Lei SHI, Rui-min LIU and Qiao-rong LIU \\ Gansu People's Hospital Stomatological Department Maxillofacial Surgery, China
}

Keywords: Vacuum sealing drainage, Facial spatial infection, Purulent core, Urokinase.

\begin{abstract}
Objective: To discuss treatment of purulent core in facial spatial infection by VSD technique and handling effect; Methods: patients with drainage tube blockage by purulent core are selected as research objects among patients undergoing treatment of oral cavity facial spatial infection by VSD technique, the formation reason and handling methods of purulent core are discussed according to clinical observation of purulent core production. Results: flushing of vomica by two groups of urokinase solution is increased everyday during whole-process treatment for each patient. The patient quantity of drainage tube blockage due to purulent core is prominent reduced according to observation. Conclusion: the function of VSD minimally invasive treatment in spatial infection treatment is greatly affected due to production of purulent core during facial spatial infection treatment by VSD technique. Flushing of infection space by urokinase solution can greatly improve VSD treatment effect.
\end{abstract}

\section{Introduction}

Minimally invasive and painless trend is gradually popularized and applied in clinical treatment with continuous development of surgical technique. Maxillofacial surgery is no exception. The author introduces vacuum sealing drainage [1] (VSD) in maxillofacial spatial infection treatment. After patient maxillofacial cellular tissues suffer from serious infection and mature vomica is formed, the vomica can be cut in the incision with the shortest distance to skin under the guidance of color doppler ultrasound, and pus can be drained. The incision can be suitably adjusted according to vomica size and vomica quantity[2]. However, the incision length is generally not larger than $1.5 \mathrm{~cm}$. When pus is basically drained completely, hydrogen peroxide solution and physiological saline can be generally adopted for flushing vomica repeatedly aiming at killing anaerobe in tissue gaps and draining more necrotic tissues. However, after hydrogen peroxide is used for flushing the vomica, a large number of strip-shaped purulent cores can be always produced. Purulent cores can be removed easily in traditional big incision open drainage[3]. However, purulent cores can always block side wall drainage port of vacuum drainage tube and drainage tube in the treatment process in minimally invasive VSD treatment, thereby seriously affecting the treatment efficiency of VSD treatment technique. The author masters the production mechanism and handling method of purulent cores in treatment through analysis and summary in order to solve the more intractable problem[4-9]. Concrete conditions are reported as follows: 


\section{Clinical Analysis}

\section{Research Object}

40 patients suffering from oral facial spatial infection and undergoing VSD method treatment from October 2013 to April 2014 are selected, and they are collected in our hospital. Inclusion criteria: (1) they conform to oral facial spatial infection diagnostic criteria; (2) they sign patient informed consent and are willing to participate in the study. Exclusion criteria: (1) patients have heart, lung, liver and renal function insufficiency; (2) patients have serious blood coagulation function disorder. These patients are divided into control group and observation group according to admission odd or even numbers. Odd number patients are included into control group, and even number patients are included into observation group. Each group is composed of 20 cases respectively. Comparison of gender, age, infection source and other general information for patients in the two groups is not statistically significant $(P>0.05)$, and the two groups are comparable[10-12].

\section{Method}

Control Group. Conventional flushing treatment with VSD flushing fluid is adopted in the control group. Concrete method is shown as follows: flushing tube and drainage tube are arranged in vomica position under $2 \%$ lidocaine anesthesias. Flushing fluid is added from the flushing tube. The flushing fluid refers to conventional hydrogen peroxide solution and physiological saline. Our department adopts intermittent flushing method 3 times a day until the tube is removed. Flushing liquid flow rate is $250 \sim 350 \mathrm{ml} / \mathrm{H}$. Effective flushing treatment time is $4 \sim 7$ days in the group.

Observation Group. The same conventional VSD flushing fluid as the control group is adopted for flushing treatment in the observation group. The difference lies in that when vacuum vomica flushing is started everyday, $50 \mathrm{ml}$ urokinase solution normal saline with concentration of $5000 \mathrm{u} / \mathrm{ml}$ is adopted for injecting into the flushing tube. Vacuum device is closed and drained for about $5 \mathrm{~min}$ during injection of urokinase solution. Urokinase solution is kept in vomica for certain period of time. Vacuum drainage system is turned on $5 \mathrm{~min}$ later. Changes in drainage liquid characters and colors at the beginning and the end are observed. If a lot of red percolate is not discovered after vacuum drainage for $1 \mathrm{~min}$, the subsequent treatment is the same as the control group. If a lot of red percolate is discovered, the vacuum drainage system is continuously closed for $10 \mathrm{~min}$, and then the vacuum system is started again. The percolate color is observed. Previous treatment is restored until the blood is rare in the drainage liquid. Effective flushing treatment time of the flushing tube in the group is 6 $\sim 7$ days.

\section{Clinical Comparative Observation}

Comparative Observation in the Treatment Process. The control group and observation group do not have special difference since the first day of treatment. However, vacuum drainage tubes are blocked by purulent core for 35 person times accumulatively in the control group. However, vacuum drainage tubes are blocked by purulent core for 13 person times in the observation group. The blocked drainage tubes should not be removed, and negative drainage tube cleaning should be implemented in order to establish new flushing drainage cycle. The vacuum suction tubes are placed in vomica again after disinfection. New vacuum drainage tubes should be replaced for 6 patients in the control group due to repeated blockage of drainage tube. Similar 
conditions are not discovered in the observation group. The treatment feeling of patients in the control group is seriously decreased compared with that of the observation group due to operation of resetting drainage tubes for many times. In addition, the subsequent recovery time can be prolonged due to unsmooth drainage of inflammatory exudate at the early stage of inflammation. Therefore, the treatment time of many patients in the control group is prominently prolonged compared with observation group. Statistics analysis is shown in Table I.

Table 1. Comparison of vacuum drainage tube blockage condition in two groups

\begin{tabular}{|c|c|c|c|c|c|c|c|c|c|}
\hline \multirow{2}{*}{ Group } & \multirow{2}{*}{$\mathrm{n}$} & \multicolumn{1}{|c|}{ Treatment duration } \\
\cline { 3 - 12 } & & 1 & 2 & 3 & 4 & 5 & 6 & 7 & Tota (person) \\
\hline Control group & 20 & 0 & 2 & 5 & 5 & 6 & 8 & 9 & 35 \\
\hline Observation group & 20 & 0 & 0 & 0 & 2 & 2 & 4 & 5 & 13 \\
\hline
\end{tabular}

Observation of Treatment Effect. The treatment effect of control group and observation group is compared. It is observed that symptoms of 18 patients in the control group are basically alleviated after treatment for 7 days. However, six patients still should be treated continuously. After observation group is treated for 7 days, symptoms of 23 patients were basically alleviated, and one patient should be treated continuously. Statistical analysis is shown in table 2.

Table 2. Clinical effect and satisfaction comparison of patients in the two groups

\begin{tabular}{|c|c|c|c|c|c|c|c|}
\hline \multirow{2}{*}{ Group } & & \multicolumn{3}{|c|}{ Clinical effect } & \multicolumn{3}{c|}{ Satisfaction } \\
\cline { 3 - 8 } & $\mathrm{n}$ & $\begin{array}{c}\text { Cure } \\
\mathrm{d}\end{array}$ & Improvd & Invalid & Satisfied & $\begin{array}{c}\text { More } \\
\text { satisfied }\end{array}$ & Dissatisfied \\
\hline Control group & 2 & 15 & 5 & 0 & 10 & 6 & 4 \\
\hline $\begin{array}{c}\text { Observation } \\
\text { group }\end{array}$ & 2 & 19 & 1 & 0 & 16 & 4 & 0 \\
\hline
\end{tabular}

\section{Experimental Analysis}

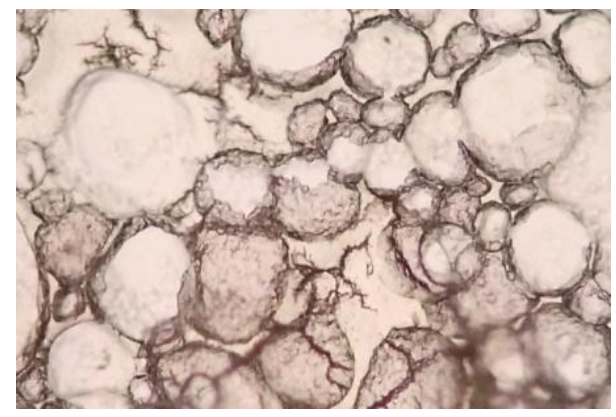

Figure 1. Image after 3\% hydrogen peroxide action on pus, and bubble colloid groups are visible;

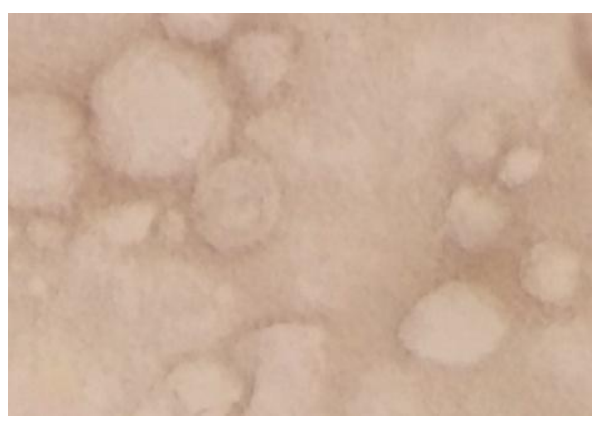

Figure $2.3 \mathrm{~min}$ after 5000 units/ml urokinase solution is added in colloid groups 


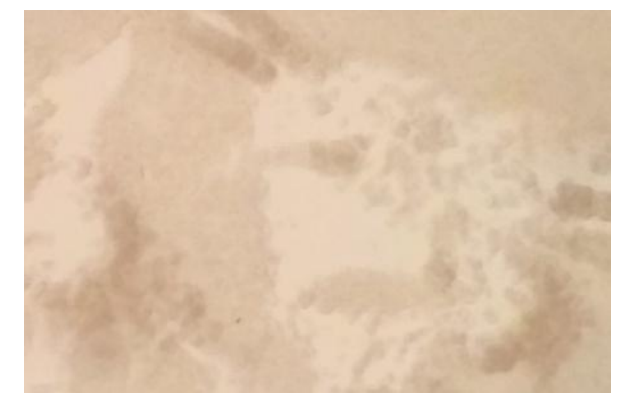

Figure 3. $3 \mathrm{~min}$ after 5000 units/ml urokinase solution is added in colloid groups

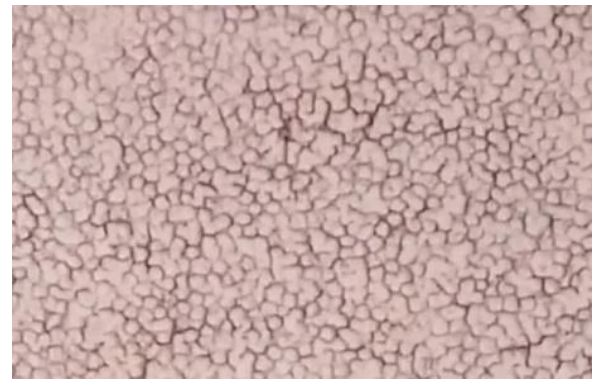

Figure $5.5 \mathrm{~min}$ after 5000 units/ml urokinase solution is added in small colloid groups

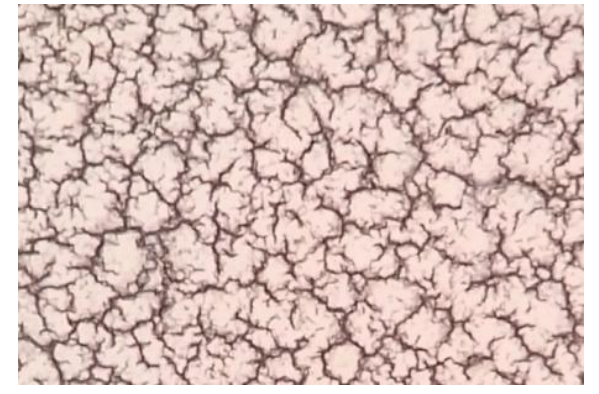

Figure 4. Smaller bubble colloid groups are visible after $1 \%$ hydrogen peroxide action on pus

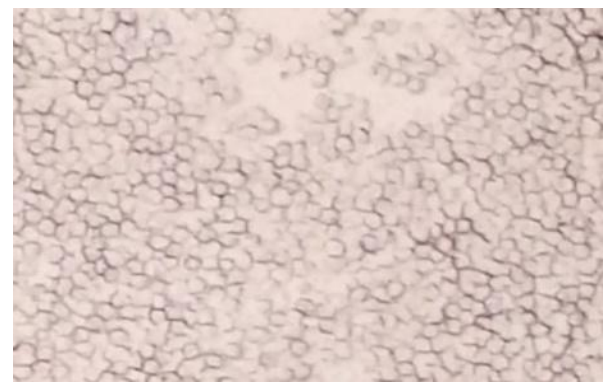

Figure 6. 5 min after 5000 units/ml urokinase solution is added in small colloid groups

When abscess is cut and vomica is flushed, the adopted flushing liquid normally contains 3\% hydrogen peroxide solution in conventional facial spatial infection treatment. After hydrogen peroxide is used, vacuum drainage tubes are always blocked. More detailed experiment observation should be implemented in order to observe the reason. In experiment, $3 \%$ hydrogen peroxide solution is added into pus removed from vomica. Changes of pus are observed under microscope as shown in figure 1. A lot of crumb structures are produced in pus, which are intertwined mutually. These are primary structure in purulent cores. $5000 \mathrm{u} / \mathrm{ml}$ urokinase solution is added in the pus with crumb structures in order to inhibit production of purulent cores as shown in figure 2 and figure 3. However, figure 3 shows that there are still a part of colloid masses which can not be completely loosened due to larger volume as a whole. These larger masses still can prominently increase the trend of purulent core formation. Therefore, $1 \%$ hydrogen peroxide is used for treatment in the experiment in order to prevent formation of larger colloid masses due to use of $3 \%$ hydrogen peroxide in vamica at the beginning of preventive treatment as shown in figure 4 . It is discovered that the colloid masses produced by pus is prominently reduced compared with colloid masses in figure 1 , which are evener. After $5000 \mathrm{u} / \mathrm{ml}$ urokinase solution is added in such colloid masses, the loosening effect of colloid masses is more prominent as shown in figure 5 and figure 6 . 


\section{Conclusion}

It is made clear that blockage phenomena can be produced more easily in VSD system without urokinase solution through clinical analysis. The VSD system of flushing with urokinase solution has more advantages in the aspects of treatment effect and patient satisfaction. The production mechanism of colloid masses in the VSD system can be further understood through in-vitro experiment, and the concentration of hydrogen peroxide in flushing fluid is reduced. The possibility of colloid mass formation can be prominently reduced.

\section{Discussion}

\section{Purulent Core Production Mechanism}

$3 \%$ hydrogen peroxide solution is conventionally used in the used flushing liquid when abscess is cut and vomica is flushed in conventional facial spatial infection treatment. $\mathrm{pH}$ value of $3 \%$ hydrogen peroxide solution is about 5.60. Main ingredients of pus in infection space include dead white blood cells and leaked interstitial fluid. The pus can undergo decomposition reaction when it contacts with hydrogen peroxide solution, namely $2 \mathrm{H}_{2} \mathrm{O}_{2} \rightarrow 2 \mathrm{H}_{2} \mathrm{O}+\mathrm{O}_{2}$. The reaction process belongs to exothermic reaction. Local temperature will rise sharply to $55^{\circ} \mathrm{C}$ or so. There are a lot of phospholipid and protein on the cell membrane of necrosis white blood cells in the pus. After the white blood cells are degraded, a lot of proteins can be released into the pus. The physical size of the proteins is about $50-200 \mathrm{~nm}$. A part of the protein is within the colloids (particle of $1 \mathrm{~nm}$ - 100nm) scope. The colloid aggregation is accelerated when the $\mathrm{pH}$ value is 4.5 to 6.0 and the temperature is $39^{\circ} \mathrm{C}-50^{\circ} \mathrm{C}$. Purulent cores are produced when the coagulation masses are increased rapidly.

\section{Modification of VSD Flushing Liquid}

After purulent core production mechanism in the VSD system is comprehended. The flushing liquid is suitably modified. The blockage possibility of VSD can be prominently reduced. The treatment effect of spatial infection VSD treatment can be improved.

Firstly, the concentration of hydrogen peroxide can be reduced. The hydrogen peroxide concentration is generally reduced from $3 \%$ to $1 \%$. Therefore, the antibacterial sterilization effects of hydrogen peroxide can be continuously exerted on one hand. The possibility of producing larger purulent cores after flushing liquid application can be prominently reduced on the other hand, thereby reducing VSD blockage risks.

Secondly, a group of urokinase solution can be added in daily flushing liquid sequence. Therefore, existing tiny purulent cores can be effectively alleviated, and VSD drainage tube can exert own role smoothly. Exudate in vomica and necrosis degradation fibrous tissues due to ischemia can be better excluded, thereby providing a better micro-environment for growth of fresh granulation tissues[13].

In summary, VSD technique application in oral and maxillofacial multi-spatial infection treatment is an innovation of minimally invasive technique in the maxillofacial surgery treatment. However, treatment of oral facial spatial infection by VSD always suffers from drainage tube blockage phenomena by purulent core. In the study, clinical analysis and experiment observation are implemented aiming at the phenomena. The concentration of hydrogen peroxide can be reduced, and urokinase solution is added for flushing vomica. VSD pipe blockage can be prominently reduced, 
treatment effect can be improved, and the technique can be clinically popularized and used.

\section{References}

[1] Ubbink DT, Westerbos SJ, Nelson EA, et al. A systematic review of topical negative pressure therapy for acute and chronic wounds. $\mathrm{Br} \mathrm{J}$ Surg, 2008, 95(6):685-692.

[2] Liang Yan, Wang Xiaoqin. Nursing of patients suffering from lower limb bone exposure after fracture with extensive soft tissue injury in treatment by closed vacuum drainage. Journal of Nursing, 2011, 18(6): 38-40.

[3] Yang Fan, Bai Xiangjun. New idea of wound treatment - vacuum closed drainage technique. Chinese Journal of Surgery 2010, 48(5): 387-389.

[4] Qi Xinwen, Wang Zhaojie, Chen Junping, etc. Application of vacuum closed drainage technique in large area skin defect necrosis infection necrosis and treatment effect. Orthopedic Biomechanics Materials AND Clinical Study, 2011, 8(2), 25-28.

[5] Tu Xiaohui. Nursing experience of treating maxillofacial and neck spatial infection by vacuum closed drainage technique combined with drug flushing. Journal of Nurses Training, 2013, 28(6):562-563.

[6] Deng Jian. Clinical analysis on treating 53 cases with oral and maxillofacial region spatial infection. Qinghai Medical Journal, 2012, 42(4), 15-16.

[7] Zhou Longnu, Cai Peipei, Dong Xiaowen, etc. Comprehensive treatment of severe oral and maxillofacial multi-spatial infection. Chinese Journal of Oral and Maxillofacial Surgery, 2006, 4(3): 198-201.

[8] Lu Jinying, Xu Yueping, Lu Yuehong, etc. Effect observation of treating stage IV and higher pressure sores by modified vacuum closed drainage combined with slough removal and tissue regeneration promotion traditional Chinese medicine lavage. Journal of Nursing, 2014, 21(21): 62-64.

[9] Qiu Weiliu. Oral and maxillofacial surgery, Beijing, People's Medical Publishing House 2008:197-198.

[10] Peterson LJ, Contemporary management of deep infactions of the neck. J Oral Maxillofac Surg, 1993, 51(3):266-231.

[11] Huang Siwei, Wang Mei, Lin Shaomang. Nursing of lower limb varicosity minimally invasive transilluminated powered phlebectomy. Chinese Journal of Practical Nursing, 2005, 6(6): 33-34.

[12] Pan Lulu, Fang Yiming, Lin Chongxiang. Clinical observation of vacuum closed drainage technique combined with drug lavage for treating maxillofacial and neck spatial infection [J]. Journal of Oral and Maxillofacial Surgery, 2012, 22(6):405-408;

[13] Yang Guiyuan, Qian Shiyin. Research progress of vacuum closed drainage technique [J]. Chinese Journal of Practical Surgery, 2010,30(2): 150. 